\title{
El plagio, su detección y como prevenirlo en la literatura médica
}

\section{Plagiarism, its detection and how to prevent it in the medical literature}

\author{
Miguel Urina-Triana $1{ }^{*}$ Md, MSc, PhD, FACC ${ }^{1}$ \\ ${ }^{1}$ Universidad Simón Bolívar, Facultad de Ciencias de la Salud. Barranquilla, Colombia \\ *Dirigir correspondencia a: murina1@unisimonbolivar.edu.co
}

\begin{abstract}
RESUMEN
Proceso Editorial

Recibido: 20102020

Aceptado: 01022021

Publicado:19 072021

DOI 10.17081/innosa.127 (C) Copyright 2021.

Urina $^{1}$

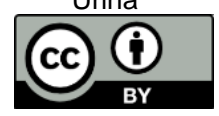

El plagio en la literatura científica médica desde antaño hasta nuestros tiempos es el peor flagelo que atenta contra la verdad que la investigación produce. Su aparición, a pesar de todos los esfuerzos por evitarlo, hace que debamos fortalecer y reflexionar sobre las medidas preventivas para desestimular esta práctica. En el presente artículo se realiza una revisión en Pubmed de las revisiones sistemáticas realizadas en los últimos 30 años para estudiar el plagio en la literatura mundial y, además, se presentan los tipos de plagio que pueden existir, sus definiciones y se pone de manifiesto cual sería la mejor forma de detectarlo, abordarlo y como implementar estrategias para prevenirlo. El control del plagio requiere de la acción combinada entre la educación, la detección y la prevención.

Resumen: Detección de plagio; mala conducta científica; falsificación.

\section{ABSTRACT}

Since ancient times, plagiarism in the medical scientific literature has been the worst scourge that threatens the truth that research produces. Its appearance, despite all efforts to avoid it, encourages to strengthen and reflect on the preventive measures to discourage this practice. In this article, a review is conducted based on the PubMed-indexed systematic reviews carried out in the last 30 years with the aim of studying plagiarism in the world literature. Also, the types of plagiarism that may exist, their definitions, and the tools and strategies for detection and prevention are presented. The control of plagiarism requires the combined action of education, detection, and prevention.
\end{abstract}

Keywords: Plagiarism detection; Scientific misconduct; Falsification.

Cómo citar: Urina M. El plagio, su detección y como prevenirlo en la literatura médica. Ciencia e Innovación en Salud. 2021. e127: 198-212 DOI 10.17081/innosa.127

Cite this dataset: Innovación en Salud, Ciencia e (2021), "Plagiarism, its detection and how to prevent it in the medical literature", Mendeley Data, V1, doi: 10.17632/3y9ks998jw.1 


\section{INTRODUCCIÓN}

El plagio es un problema muy común y complejo en la escritura científica que ha existido y transcendido desde hace mucho tiempo en la historia. Hace más de 400 años en la lengua inglesa apareció el término plagio en el mismo sentido que hoy lo usamos y como resultado de los enfrentamientos que por derecho de la autoría real de escritos hubo entre William Shakespeare y sus pares $(1,2)$. Sin embargo, es al dramaturgo Ben Jonson a quién se le ha dado el crédito de haber acuñado la palabra plagio descrita por él como "algún tipo de robo literario" (1).

A veces el plagio alcanza el status del "crimen perfecto" (그): si no es detectado y descubierto, no existe. Una de las peores excusas cuando se descubre al "ladrón" es que este siempre dice "no me di cuenta", "no quise hacerlo", "jamás tuve esa intención", "usaron mi nombre sin mi autorización", entre otros. En este orden, la intertextualidad, la cita, la auto citación y la paráfrasis son algunas excusas del escritor que lo comete. Escribir no es fácil y siempre se hace necesario tener en cuenta lo que otros han escrito, por eso es muy importante conocer como referenciar en forma apropiada. Por lo tanto, lo que se debe calificar en el plagio es la intencionalidad (4). Por consiguiente, es el autor el responsable de éste, porque a conciencia o por omisión sabe que él no es el dueño de esa idea o de esos datos.

Aunque muchas ideas son universales, es la forma de expresarlas los que las hace únicas de cada autor y es lo que se conoce con el nombre de originalidad. Muchos escritores traducen textos en forma libre no ajustándose al original con exactitud, aduciendo que es para que el lector tenga un mejor entendimiento. Esta paráfrasis que utiliza el lenguaje propio e individual para lograr una mejor comprensión del texto original constituye un hallazgo muy frecuente y que encierra la necesidad de una cita apropiada de la fuente por parte del autor pues sino se convierte inmediatamente en plagio.

En el 2015, los editores de la Revista Bioética hacen una reflexión sobre lo publicado por Spinak, quién expuso las maneras de cómo engañar a los controles de softwares anti-plagios automatizados (므). Este hecho hace un llamado de atención que, si bien es un paso muy importante implementar controles automatizados, también es necesario que el autor sea consciente que es su responsabilidad es evitar a toda costa el plagio. La revista pone de manifiesto un problema aún no resuelto para evadir el control automatizado y que textualmente dice así (므, $\underline{6})$ :

"Para superar la revisión sistemática de los programas de control, es suficiente utilizar un procedimiento casi hecho a mano:

1.Pegue el texto de su artículo y proceda al control de plagio con uno de los programas clásicos (iThenticate, Turnitin, Urkund, o cualquier otro).

2. El resultado será un documento con una serie de observaciones en algunas partes de su texto reconocidos como plagio.

2.1. Modifique los párrafos marcados como sospechosos cambiando el orden de las frases, haciendo paráfrasis o utilizando sinónimos. 
2.2. Otro procedimiento consiste en traducir el texto en otro idioma utilizando Google y volver a la traducción. Por ejemplo, escriba en inglés, traduzca al portugués, luego al español, y finalmente de vuelta al inglés.

3. Vuelva al paso (1) hasta que el texto vuelva sin observaciones.

4. Si no hay comentarios, su texto no se considerará plagio por los programas de control" (ㅁ).

Esto demuestra que "hecha la ley, hecha la trampa". Se requiere por ello un compromiso ético del autor, quién debe estar verdaderamente consciente de los alcances de su actuación y de la responsabilidad que conlleva acreditarse escritos o ideas que no le pertenecen. A pesar de las crecientes técnicas de control que existen, estas no son suficientes si los autores no se hacen responsables de revisar sus escritos para evitar el plagio.

Una revisión en Medline de todas las publicaciones retractadas en 42 años desde enero de 1966 a febrero de 2008 encontró que de las 213 publicaciones retractadas el $41.8 \%$ fueron retractadas por plagio; el $52.1 \%$ por falsificación o fabricación falsa; el $2.3 \%$ por disputas entre autores por problemas éticos y el $1.4 \%$ por causas desconocidas (ㄱ). Pero lo más relevante del artículo fue la asociación que se encontró entre la publicación retractada y el origen del primer autor afiliado cuya procedencia generalmente era de países de bajos ingresos económicos. Alfaro-Toloza y colaboradores en una carta al editor publicada en 2013 en la revista Current Medical Research and Opinion, y tomando como ejemplo la revisión hecha por Stretton7 llama la atención sobre la necesidad de investigar en Latinoamérica la mala conducta y el plagio en las publicaciones (ㅇ).

Una publicación encontró en Pubmed una proporción de retractaciones en 611 revistas de 2,5 por cada 10.000 publicaciones entre enero 1 de 2013 a diciembre 31 de 2016, con una diferencia significativa en lo que respecta a la aparición de un mayor número de éstas en las revistas de menor factor de impacto $(73 \%)$ comparada con las de mayor impacto $(61 \%)$ con una $p=0.0019$. Según la categoría de clasificación del Journal Citation Report también fueron observadas diferencias en el porcentaje de retractaciones debidas a mala conducta $(p<0,001)(\underline{9})$.

En 2017 una revisión de publicaciones retractadas en neurocirugía incluyó 97 publicaciones con un factor de impacto entre 0.57 a 35.03 , la mayoría $(n=61)$ fueron retractados en los últimos 5 años y de ellos 22 (22,6\%) lo fueron por plagio (10).

Un informe publicado en 2018 llama la atención sobre el incremento del plagio en la literatura médica en las últimas dos décadas y que es atribuido por sus autores al mayor y fácil acceso a través de los medios electrónicos a vastas fuentes de información (11). Se dice que el primer artículo acusado de plagio en la misma fue publicado en 1979 (12).

También en 2018 una publicación realizada sobre el análisis de las retractaciones en las revistas de acceso abierto (en inglés "Open Access") llama la atención sobre el incremento de las mismas desde 2010 y mostró que en 621 publicaciones retractadas las causas de dicha retractación fueron:148 por error; 142 por plagio; 101 por publicación duplicada; 98 por sospecha de fraude o fraude y 93 por revisión de pares inválida. La mayoría de estas se observaron en las revistas de bajo impacto y los países con más retractaciones fueron China, India, Irán y Estados Unidos principalmente (13). 
El 25 de octubre de 2018, Iván Oransky, presentó una iniciativa con la creación de una base de datos de acceso abierto y gratis para el estudio de las retractaciones en la literatura científica14. Al utilizar este software fue posible encontrar 49 casos de artículos retractados para Colombia en el período comprendido entre 01 de enero de 1990 a 10 de abril de 2021 de los cuales el $55 \%$ $(n=27)$ fueron por plagio (14).

En 2020, Mousavi y colaboradores (15) en una revisión en varias bases de datos como Google Scholar, Web of Science, Scopus, PubMed, Nature Index, Publication Ethics and Retraction Watch de los últimos 10 años encontraron que el $65.3 \%$ de los artículos retractados por mala conducta la mayoría eran por plagio. También en 2020, Nair y colaboradores (16), realizaron un análisis de la razón de 350 artículos retractados en el área de anestesiología y la causa más común fue el fraude en el $49,4 \%$ de ellos.

El objetivo de este artículo es presentar una revisión realizada en Pubmed en un período de 30 años sobre cuales han sido las revisiones sistemáticas realizadas sobre el plagio en la investigación biomédica y además presentar cuales son las definiciones existentes del plagio en la literatura médica, cuales han sido las mejores formas de detectarlo y como se podrían plantear las mejores estrategias para prevenirlo en nuestro contexto universitario.

\section{MÉTODOS}

Se realizó una búsqueda bibliográfica en PubMed con los siguientes términos MeSH y distractores Booleanos: "Plagiarism" AND "Biomedical Research". Se utilizaron los siguientes filtros de selección de los artículos: 1) Publicados desde 01/01/1990 a 31/12/2020; 2) De texto completo; 3) En idioma Inglés y Español y 4. Según tipo de artículo: Revisión sistemática ("Filters applied: Full text, Systematic Review, English, Spanish, from 1990/1/1 - 2020/12/31") (Tabla 1). En los artículos encontrados se buscó información en ellos sobre definición, detección, abordaje o prevención del plagio.

Tabla 1. Criterio de búsqueda utilizado en Pubmed

\begin{tabular}{|c|c|c|c|c|c|}
\hline $\begin{array}{l}\text { Número } \\
\text { de } \\
\text { búsqueda }\end{array}$ & Consulta & \begin{tabular}{|l|} 
Ordenado \\
por
\end{tabular} & Filtros & Detalles de la búsqueda & Resultados \\
\hline 1 & $\begin{array}{l}\text { "Plagiarism" } \\
\text { AND } \\
\text { "Biomedical } \\
\text { Research" }\end{array}$ & \begin{tabular}{|l|} 
Publication \\
Date
\end{tabular} & $\begin{array}{l}\text { Full text, } \\
\text { Systematic } \\
\text { Review, } \\
\text { English, } \\
\text { Spanish, } \\
\text { from } \\
1990 / 1 / 1- \\
2020 / 12 / 31\end{array}$ & $\begin{array}{l}\text { ("Plagiarism"[All Fields] AND } \\
\text { "Biomedical Research"[All Fields]) } \\
\text { AND ((systematicreview[Filter]) AND } \\
\text { (fft[Filter]) AND } \\
\text { (1990/1/1:2020/12/31[pdat]) AND } \\
\text { (english[Filter] OR spanish[Filter])) }\end{array}$ & 3 \\
\hline
\end{tabular}

Fuente: Elaboración propia

\section{RESULTADOS}

La búsqueda realizada arrojó un total de tres revisiones sistemáticas en los últimos 30 años. (Tabla 2). Estas tres revisiones sistemáticas se hicieron en el idioma inglés, la primera de ellas 
publicada en la Cochrane Library en 2016, fue realizada por Marusic y Colaboradores (17) y evaluó la efectividad de las intervenciones educativas o políticas en la integridad de la investigación o la realización responsable de la investigación sobre el comportamiento y las actitudes de los investigadores en salud y otras áreas de investigación.

Los autores delimitaron el periodo de búsqueda a los artículos publicados entre 1990 a 2014 y arrojó 31 estudios todos publicados en inglés con 9571 participantes, descritos en 33 artículos $(\underline{17})$.

Los autores concluyeron que la base de evidencia relacionada con las intervenciones para mejorar la integridad de la investigación era incompleta y los estudios que se habían realizado eran heterogéneos, inapropiados para los metaanálisis y su aplicabilidad a otros contextos y poblaciones es incierta. Muchos estudios tuvieron un alto riesgo de sesgo debido a la elección del diseño del estudio y las intervenciones a menudo se informaron de manera inadecuada. Incluso cuando se utilizaron diseños aleatorios, los resultados fueron difíciles de generalizar. Debido a la muy baja calidad de la evidencia, los efectos de la capacitación en la realización responsable de la investigación sobre la reducción de la mala conducta en la investigación son inciertos. La evidencia de baja calidad indica que la capacitación sobre plagio, especialmente si involucra ejercicios prácticos y el uso de software de comparación de texto, puede reducir la ocurrencia de plagio (17).

La segunda revisión sistemática, realizada en 2019 por Yi y colaboradores (18), presentó una serie de estudios empíricos publicados sobre la integridad de la investigación y la mala conducta en la investigación biomédica en China. El objetivo fue obtener una visión integral de la investigación existente realizada en China sobre la conciencia, las actitudes, las percepciones y las experiencias de los investigadores biomédicos con respecto a la integridad y la mala conducta de la investigación.

De una revisión inicial de 1578 estudios solo de artículos de revistas publicados entre mayo y junio de 2016, se seleccionaron 21 estudios de los cuales: 2 estudios utilizaron métodos cualitativos (entrevistas) y (19) utilizaron métodos cuantitativos (cuestionarios). Los investigadores encontraron que se habían realizado una gran cantidad de estudios de integridad de la investigación en China, pero la evidencia empírica aún es limitada.

China es uno de los países con mayor producción de publicaciones científicas. Sin embargo, al igual que muchos otros países y regiones, la investigación científica china se ve amenazada por la mala conducta de la investigación, cuya existencia y daño han sido reconocidos por China y las comunidades científicas internacionales. Los autores concluyeron que el fortalecimiento de la formación en integridad de la investigación, el desarrollo del sistema de gobernanza y la mejora del sistema de evaluación científica fueron áreas de especial atención en varios estudios. Esta revisión demostró que se ha dedicado un número sustancial de artículos a la integridad de la investigación en China, pero solo unos pocos estudios proporcionaron una evidencia diferente a la empírica y por esta razón concluyen que sería crucial realizar más investigaciones para explorar las percepciones en profundidad de los investigadores y evaluar los cambios (18). 
La tercera revisión sistemática realizada en 2019 por Stavale y colaboradores, que como objetivo tuvo el investigar el perfil de las retractaciones de la investigación médica y en ciencias de la vida de autores afiliados a instituciones académicas brasileñas (19). Los autores seleccionaron los avisos de retractación publicados desde enero de 2004 hasta agosto de 2017 sobre artículos que tenían al menos un autor afiliado a una institución brasileña, independientemente de su cargo de autor e independientemente del año de publicación del artículo original.

De 3179 artículos encontrados al final de la búsqueda, se obtuvo una muestra final de 65 artículos de 55 revistas diferentes que cumplieron los criterios de selección, con factores de impacto informados que iban desde 0 a 32,86, con un valor medio de 4,40 y una media de 4,69. Los tipos de documentos encontrados fueron fe de erratas, artículos retractados, artículos retractados con aviso de retractación, avisos de retractación con fe de erratas y avisos de retractación. La evaluación del sitio web Retraction Watch agregó 8 artículos que no habían sido identificados por la estrategia de búsqueda utilizando las bases de datos bibliográficas. Las publicaciones retractadas cubrieron una amplia gama de diseños de estudios. Algunos artículos se retiraron por al menos dos razones distintas. Entre los artículos estudiados, el plagio fue el principal motivo de retractación (60\%). Se encontraron datos faltantes en el $57 \%$ de los avisos de retractación, lo que fue una limitación para esta revisión. Además, como dato curioso, el $63 \%$ de los artículos fueron citados tras su retractación.

Este estudio enfatizó la importancia de la acción coordinada entre todos los involucrados en la producción científica para promover la transparencia de la investigación. Hay un impacto positivo de las buenas prácticas al realizar investigaciones e informar y publicar avisos de retractación. Los factores subyacentes que involucran la mala conducta en la investigación siguen sin estar claros. Las medidas para prevenir la mala conducta pueden tomar en consideración las particularidades de cada sociedad, incluidas las debilidades y fortalezas, dependiendo de los aspectos culturales. Sin embargo, el impacto de la mala ciencia no tiene fronteras y no depende de la cultura (19).

\section{DISCUSIÓN}

En nuestra revisión realizada en un período de 30 años en Pubmed no encontramos muchas revisiones sistemáticas que abordaran el tema de plagio en la literatura de la investigación biomédica. Sin embargo, las tres revisiones encontradas reúnen un total de 117 publicaciones, con resultados que hablan de la magnitud del problema que el plagio original.

En estas tres revisiones demuestran primero, que las prácticas inadecuadas y la conducta no profesional en la investigación clínica desperdician una parte significativa de los fondos de atención médica y perjudican la salud pública y segundo que la proporción o porcentaje de plagio encontrado como causal de retractación es alta, producto del mal comportamiento en la investigación y que llama la atención sobre la necesidad de controlarlo.

El control del plagio requiere por lo tanto de las siguientes acciones de: 1. Educación, 2. Detección y 3. Prevención.

En el primero, las acciones en educación, se hace necesario que desde la formación del estudiante se analicen estrategias educativas para darle a conocer al estudiante todo el 
conocimiento de cómo realizar adecuadamente la escritura científica, como usar las citas en forma correcta, cuál es la definición del plagio y que tipos de plagio están presentes en la literatura biomédica. Por qué el conocerlos de una manera profunda, hará que se minimice la posibilidad de cometerlo.

Tabla 2. Artículos encontrados en Pubmed de acuerdo con el criterio de selección propuesto $(\mathrm{N}=3)$

\begin{tabular}{|c|c|c|c|c|}
\hline Año & Título & Autores & $\begin{array}{l}\text { Artículos } \\
\text { revisados }\end{array}$ & $\begin{array}{l}\text { Observaciones } \\
\text { sobre los } \\
\text { Resultados }\end{array}$ \\
\hline 2016 & $\begin{array}{l}\text { Interventions to prevent misconduct and } \\
\text { promote integrity in research and } \\
\text { publication. Cochrane Database Syst Rev. } \\
2016 \text { Apr 4;4(4):MR000038. doi: } \\
\text { 10.1002/14651858.MR000038.pub2. } \\
\text { PMID: 27040721; PMCID: PMC7149854. }\end{array}$ & $\begin{array}{l}\text { Marusic } A, y \\
\text { colaboradores }^{17} \text {. }\end{array}$ & 31 & $\begin{array}{l}21 \text { estudios } \\
\text { evaluaron los efectos } \\
\text { de las intervenciones } \\
\text { relacionadas con el } \\
\text { plagio y } 10 \text { estudios } \\
\text { evaluaron las } \\
\text { intervenciones en la } \\
\text { integridad / ética de la } \\
\text { investigación. }\end{array}$ \\
\hline 2019 & $\begin{array}{l}\text { Integrity in Biomedical Research: A } \\
\text { Systematic Review of Studies in China. Sci } \\
\text { Eng Ethics. } 2019 \text { Aug;25(4):1271-1301. } \\
\text { doi: 10.1007/s11948-018-0057-x. Epub } \\
\text { 2018 May 2. PMID: } 29721845 .\end{array}$ & $\begin{array}{l}\mathrm{Yi} \quad \mathrm{N}, \quad \mathrm{y} \\
\text { colaboradores }^{18} \text {. }\end{array}$ & 21 & $\begin{array}{l}\text { La mayoría de los } \\
\text { participantes en } \\
\text { estos } 21 \text { estudios } \\
\text { informaron que la } \\
\text { integridad de la } \\
\text { investigación es de } \\
\text { gran importancia y } \\
\text { que obedecen las } \\
\text { normas académicas } \\
\text { durante su } \\
\text { investigación. }\end{array}$ \\
\hline 2019 & $\begin{array}{l}\text { Research misconduct in health and life } \\
\text { sciences research: A systematic review of } \\
\text { retracted literature from Brazilian } \\
\text { institutions. PLoS One. } 2019 \text { Apr 15;14(4): } \\
\text { e0214272. } \\
\text { 10.1371/journal.pone.0214272. PMID: } \\
\text { 30986211; PMCID: PMC6464327. }\end{array}$ & $\begin{array}{l}\text { Stavale } \mathrm{R}, \quad \mathrm{y} \\
\text { colaboradores }^{19} \text {. }\end{array}$ & 65 & $\begin{array}{l}\text { Hubo } 39 \text { estudios } \\
(60 \%) \text { retractados. } \\
\text { Los estudios } \\
\text { experimentales y las } \\
\text { revisiones de la } \\
\text { literatura fueron el } \\
84,6 \% \text { de los } \\
\text { artículos retractados. }\end{array}$ \\
\hline TOTAL & & & 117 & \\
\hline
\end{tabular}

Fuente: Elaboración propia

\section{Para abordar la definición y sus tipos revisamos lo siguiente:}

Cómo se define el plagio y cuáles son los tipos existentes de plagio en la literatura médica Según el diccionario de la Real Academia Española, la palabra plagiar proviene del latín plagiāre que significa "copiar en lo sustancial obras ajenas, dándolas como propias" (20). Se podría definir como plagio cuando alguien presenta como suyo el trabajo publicado o no publicado de otros, incluidas ideas, texto académico, imágenes, diseño de investigación y datos, como nuevo y original en lugar de acreditar la fuente existente (21). Valpy, en 1828 en 
su diccionario etimológico de la lengua latina lo define como "el crimen de secuestrar" (22). Los tipos de plagio más frecuentes que se pueden encontrar en la literatura médica (23-26) se presentan resumidos en la Tabla 3.

Tabla 3. Tipos de plagio frecuentes en la literatura médica

\begin{tabular}{|c|c|}
\hline Tipo & Definición \\
\hline $\begin{array}{l}\text { Plagio no } \\
\text { intencional o } \\
\text { involuntario }\end{array}$ & $\begin{array}{l}\text { Cuando se comete plagio no intencional o involuntario al } \\
\text { parafrasear un contenido y no citar o agregar créditos. }\end{array}$ \\
\hline Plagio intencional & $\begin{array}{l}\text { Cuando alguien se atribuye una idea o palabras de otra persona } \\
\text { como si fueran propias, pero sabe y es consciente de que está } \\
\text { realizando una acción incorrecta. }\end{array}$ \\
\hline Plagio por pago & $\begin{array}{l}\text { Cuando se contrata a otra persona o a una empresa para que } \\
\text { realice el trabajo o se compra un trabajo realizado por otro y se } \\
\text { pone como si se hubiera hecho como propio. }\end{array}$ \\
\hline Plagio total & Cuando se copia en forma completa una obra ya publicada. \\
\hline Plagio de una idea & $\begin{array}{l}\text { El utilizar la misma idea, pensamiento o invención aun sin copiar } \\
\text { ninguna palabra o frase del articulo original y se presenta como } \\
\text { propia sin dar el reconocimiento, lo mismo puede equivaler a plagio. }\end{array}$ \\
\hline $\begin{array}{l}\text { Plagio palabra por } \\
\text { palabra }\end{array}$ & $\begin{array}{l}\text { Cuando un texto o contenido se copia exactamente como está } \\
\text { escrito sin ningún tipo de crédito o cita }\end{array}$ \\
\hline $\begin{array}{l}\text { Plagio tipo } \\
\text { mosaico }\end{array}$ & $\begin{array}{l}\text { Copiar el contenido de diferentes sitios web o fuentes y } \\
\text { combinarlos sin ningún tipo de crédito o cita. }\end{array}$ \\
\hline Plagio global & $\begin{array}{l}\text { Esto significa reclamar deliberadamente todo el trabajo de otra } \\
\text { persona como propio. }\end{array}$ \\
\hline Auto plagio & $\begin{array}{l}\text { Esto es cuando vuelve a enviar un trabajo propio publicado } \\
\text { previamente como si fuera reciente. Es decir, cuando se utiliza el } \\
\text { mismo texto en un nuevo documento sin citar el trabajo previo u } \\
\text { original }\end{array}$ \\
\hline Citación incorrecta & $\begin{array}{l}\text { Citar a un autor en forma textual pero no se coloca el texto entre } \\
\text { comillas. }\end{array}$ \\
\hline $\begin{array}{l}\text { Parafraseo } \\
\text { inadecuado }\end{array}$ & $\begin{array}{l}\text { Cuando al citar y parafrasear las ideas de un autor se cambia el } \\
\text { sentido o se saca de contexto la idea original }\end{array}$ \\
\hline $\begin{array}{l}\text { Plagio de } \\
\text { traducción }\end{array}$ & $\begin{array}{l}\text { En el que un texto de otro idioma se traduce literalmente y luego se } \\
\text { usa sin marcar como traducción y se cita en un artículo científico. } \\
\text { También es un plagio de traducción, si la traducción no es literal, } \\
\text { sino "sólo" en analogía. }\end{array}$ \\
\hline $\begin{array}{l}\text { Plagio de gráficas, } \\
\text { de figuras y de } \\
\text { tablas, imágenes, } \\
\text { fotos, diagramas y } \\
\text { similares. Copias } \\
\text { no autorizadas }\end{array}$ & $\begin{array}{l}\text { Cuando se toman imágenes, fotos, diagramas y similares y se } \\
\text { utilizan sin la autorización o permiso del autor y/o editor que las } \\
\text { hizo. }\end{array}$ \\
\hline
\end{tabular}

Fuente: Elaboración propia 
Empresas productoras software de anti plagios como la empresa iThenticate ha realizado una revisión sobre los diez tipos más frecuentes de plagio y de malas prácticas (27). Turnitin también presentó un infografía con las 12 formas de trabajo no original (28).

Con relación a las acciones de detección y prevención del plagio en la literatura biomédica se revisó lo siguiente:

\section{Forma de detectar el plagio y como abordarlo que estrategias existen para prevenirlo}

En 1977 fue establecido el Comité de Ética en Publicaciones (COPE) por un pequeño grupo de revistas médicas en Inglaterra y en la actualidad agrupa aproximadamente 7000 miembros. COPE ha definido 16 estándares mínimos éticos que se debe cumplir en una revista, los cuales están basados en los principios de transparencia para poder ayudar a los editores a implementar flujogramas que puedan ayudar a sospechar la presencia de plagio en un manuscrito y qué hacer si se sospecha plagio en un manuscrito sometido (29).

En 1982 se fundó la Asociación Europea de Editores para la Ciencia (EASE), que también ha producido 15 puntos de recomendación a los autores (스) $)$.

Teniendo en cuenta las limitaciones y confiabilidad del software de detección, el plagio se resuelve mejor concentrándose en el sistema educativo de prevención. Muchas instituciones han comenzado a implementar cursos que enseñan conducta responsable en la investigación y discuten abiertamente una serie de problemas en la revisión actual de la integridad de la investigación. Aunque este es un paso en la dirección correcta, estos programas educativos se ofrecen actualmente a una fracción de investigadores, mientras que muchos otros investigadores, tanto con experiencia como sin experiencia, operan sin dicha instrucción. La integridad en la investigación debe ser un valor incorporado en la educación continua y debe apuntar a todos los investigadores (1ㅜ).

Los estudiantes, investigadores novatos y algunos profesores no tienen una claridad sobre qué es lo que constituye un plagio y cuál es la diferencia entre el índice de similitud vs. el plagio (en inglés "similitary índex" vs. "plagiarism")(12). En un encuesta realizada entre estudiantes universitarios el $84.4 \%$ de ellos no fue capaz de distinguir entre uno y el otro (르).

Un estudio mostró que entre el $20-25 \%$ de las citas, se citan incorrectamente en la literatura médica (33), hay errores de contendido (fácticos) o de fuente (citas indirectas incorrectas). Los errores de contenido se dividieron en mayores o menores según el grado en que la información difería de la original. El tanto por ciento de coincidencia con otros textos nunca va a ser un criterio irrefutable para determinar si hay o no plagio.

La detección del plagio con frecuencia se hace a través de herramientas específicamente elaboradas para ello. Algunos ejemplos de estas herramientas para identificar similitudes son ArticleChecker, CrossCheck/iThenticate, Grammarly, PlagiarismChecker, TurnltIn, Viper entre otras (34).

Todos los softwares anti-plagios del mundo cuentan con bases de datos que aglutinan a miles de millones de fuentes, tales como repositorios de revistas científicas y tesis doctorales, entre otros. La correcta elección del software a utilizar es muy importante para lograr una buena verificación de similitud ( $\underline{35})$ Existen opiniones diversas que indican que si el índice de similitud es superior al 15 o al $20 \%$ podría entonces tratarse de plagio (ㅌ6), sin embargo algunas 
instituciones universitarias no utilizan umbrales de plagio cuando se utiliza una de estas herramientas (푸).

La prevalencia de fraude especialmente sobre la invención de datos y manipulación de datos es difícil de medir así como también lo es el control de duplicados de ahí la importancia del uso de herramientas de detección de similitud como una costumbre antes de someter a publicación (요).

Es muy importante tener bien claro que después de correr el manuscrito en la herramienta detectora escogida hay que tomarse el trabajo de revisar una por una las similitudes encontradas en forma metódica y ordenada, para poder establecer o no la comisión de un plagio. Aquí es donde juega un papel muy importante el profesor o educador ante el estudiante, ya que este en forma consciente y consistente deberá al revisar un documento ser capaz de establecer la originalidad que este tiene.

Como ocurre con la mayoría de las cosas, la prevención todavía tiene muchas ventajas sobre las medidas curativas ( $\underline{39})$,y aunque las razones para explicar su incremento son muy complejas, existe una causa que si se puede mejorar en el contexto de nuestras Universidades en Colombia.

Se requiere trabajar por ello, en proveer una mayor educación entre los estudiantes de pre y postgrado así mismo en los profesores para trabajar en la gestión del conocimiento de lo qué es y lo que no es plagio y con esto poder sembrar una cultura anti-plagio universitaria donde el estudiante deberá aprender a identificar como se debe citar correctamente en la literatura médica.

Explorar por tanto en los estudiantes y los académicos la actitud ante el plagio es un primer paso para la lucha contra este comportamiento (누). Esta exploración debe hacerse en forma sistemática y periódica.

Las conversaciones sobre conducta responsable en la investigación deben ir más allá del "crimen" de fabricación, falsedad y plagio para hablar sobre cómo las conductas de mala conducta pueden socavar los esfuerzos de investigación y desestabilizar las cualidades mismas de validez y precisión que son tan vitales para el campo de la investigación, la ciencia y la medicina. La instrucción sobre la prevención del plagio debe comenzar con los principios de la escritura ética (31).

Chowman y colaboradores (41), recomendaron los siguientes consejos para evitar el plagio:

1. Siempre se debe reconocer la fuente original de ideas, texto o ilustraciones.

2. Si el texto se toma literalmente, debe ir entre comillas.

3. Una idea que ha sido reinterpretada por el texto de autor que ha sido parafraseado aún debe reconocer la fuente original.

4. Si el autor no está seguro de si la idea o el hecho es de conocimiento común, es mejor, citar la fuente.

5. Si un fragmento de texto se toma de un trabajo anterior propio, debe indicarse claramente.

6. Se requiere permiso por escrito para usar borradores, datos, imágenes, etc. publicados anteriormente. 
7. En cualquier momento después de enviar el manuscrito o después de su publicación, si el autor descubre que ha usado involuntariamente las ideas o el texto de otra persona sin las debidas referencias, debe informar inmediatamente al editor de la revista y pedir consejo.

Además de estas, se podría añadir una octava recomendación, la cuál sería la responsabilidad que tiene el autor de realizar siempre una búsqueda de plagio en su trabajo, de manera que antes de someterlo a publicación, haya reconocido cualquier similitud con cualquier otro escrito. Esto debería considerarse como una buena práctica habitual, sistemática y metódica.

Muchas instituciones y organizaciones tienen políticas específicas para resolver estos temas de plagio(42-46). Un ejemplo de estas, la constituyen las políticas adoptadas por el comité de publicaciones del Optical Society of América (OSA)(47). Su junta de editores, al descubrir un plagio, hace que el editor encargado de la revista realizar una investigación preliminar sobre las acusaciones y se le pedirá una explicación al autor acusado del mismo. Si hay justificación para una acción adicional el editor enviara el caso al panel de revisión de ética de la editorial OSA, quién utilizará las pautas trazadas por instituciones que ya hemos mencionado (COPE y $E A S E$. El castigo impuesto va desde una simple advertencia, hasta la divulgación de su nombre en una "lista negra" donde se prohibirán sus futuras publicaciones.

El comité editorial de cualquier revista tendrá que estar muy pendiente y vigilante del marco legal en cómo se conduce la investigación médica y particularmente cuando existan grupos poblacionales donde esta exigencia sea mucho mayor como es el caso de la investigación en sujetos pediátricos donde el diseño, la conducción y el reporte de los resultados deben realizarse con la más alta calidad científica (48). Las sanciones estarán en relación con la naturaleza de la violación y generalmente estarán determinadas por el alcance del plagio, por ejemplo, el auto plagio se considera menos grave que el plagio del contenido de otros.

Existe un problema evidente de plagio en los países en desarrollo donde la reforma educativa actual permite lagunas en la integridad académica, incluido el gran impulso del plagio de copiar y pegar (en inglés "copy and paste") (노).

Por último, independientemente de las sanciones académicas a las que fuera acreedor el autor que plagia, no menos importante es lo que este tiene que saber sobre las implicaciones legales a lo que puede verse sometido desde el punto de vista jurídico al cometer dicha acción ( $\underline{50}$ ). En los artículos 270 al 272 del Código Penal Colombiano, se presentan las normas relativas a la protección de los derechos de autor ( $\underline{51})$.

Las instituciones científicas y académicas deberían tener siempre una unidad de seguimiento, investigación y desarrollo de la calidad de las publicaciones (르) e incorporar dentro de su plan educativo docente una forma metodológica, sistemática y continua de enseñanza del porqué, el cómo evitar el plagio y las consecuencias que este trae (흐).

\section{CONCLUSIONES}

1. El control del plagio requiere de la acción combinada entre la educación, la detección y la prevención, junto con la imposición de sanciones al plagiario. Con la interacción de ellas es que podemos esperar una reducción significativa de las incidencias de plagio y lograr con ello una integridad científica. 
2. Sólo aprendiendo la conducta de investigación adecuada y cuáles son las mejores prácticas de redacción podrá ser posible que los estudiantes aprendan a preservar la integridad académica evitando el plagio. Se requiere fomentar educación sobre la forma correcta de realizar la escritura científica y cuál debe ser la estrategia para tener éxito al hacerla tanto en español como en inglés (ㅁ4).

Contribución de autores: Miguel Urina-Triana, es el único autor de la revisión narrativa. Fue el encargado de la Conceptualización, Metodología y Escritura del artículo.

Fondos: Esta investigación no recibió fondos externos.

Conflictos de intereses: Ningún conflicto de intereses por declarar.

Información acerca del autor: Director del programa de especialización en Cardiología, Facultad de Ciencias de la Salud, Universidad Simón Bolívar-Barranquilla; Director de la Fundación del Caribe para la Investigación Biomédica; Board Editor of BMC Cardiovascular Disorders; Editor de Sección Revista Colombiana de Cardiología y Cirugía Cardiovascular; Board Editor de la Revista Argentina de Insuficiencia Cardiaca; Miembro del Comité Consultivo Científico Revista Colombiana de Nefrología; Reviewer of the Journal of Medical Case Report.

\section{REFERENCIAS}

1. Vinod KR, Banji D. Plagiarism- history, detection and prevention. Pharm News. 2011;3(1):1-4.

2. Moss S. A history of plagiarism (not my own work) [Internet]. 2021. https://www.theguardian.com/books/2005/nov/23/comment.stephenmoss

3. Ruiz Casanova JF. Plagio y traducción, o la traducción como plagio. 1611 Rev Hist La Traducción. 2017;(11):1-9. https://raco.cat/index.php/1611/article/view/333525

4. Das N. Intentional or unintentional, it is never alright to plagiarize: A note on how Indian universities are advised to handle plagiarism. Perspect Clin Res. 2018;9(1):56-7. DOl: 10.4103/picr.PICR 14017

5. Los Editores. El plagio, la copia, la imitación: una reflexión cada vez más indispensable. Rev Bioética [Internet]. $2015 \quad$ Apr;23(1):9-11. http://www.scielo.br/scielo.php?script=sci_arttext\&pid=S1983$80422015000100009 \& \operatorname{lng}=$ pt\&tlng=pt

6. Spinak E. Ética editorial: as arbitragens fraudulentas [Internet]. 2015 [cited 2020 Oct 15]. https://blog.scielo.org/blog/2015/02/20/etica-editorial-as-arbitragens-fraudulentas/\#. VO23M_nF-Y1

7. Stretton S, Bramich NJ, Keys JR, Monk JA, Ely JA, Haley C, et al. Publication misconduct and plagiarism retractions: A systematic, retrospective study. Curr Med Res Opin. 2012;28(10):1575-83. DOI: 10.1185/03007995.2012.728131

8. Alfaro-Toloza P, Mayta-Tristan P, Rodriguez-Morales AJ. Publication misconduct and plagiarism retractions: A Latin American perspective. Curr Med Res Opin. 2013;29(2):99-100. DOI: 10.1185/03007995.2012.755504

9. Campos-Varela I, Villaverde-Castañeda R, Ruano-Raviña A. Retraction of publications: a study of biomedical journals retracting publications based on impact factor and journal category. Gac Sanit. 2020;34(5):430-4. DOI: 10.1016/j.gaceta.2019.05.008 
10. Wang J, Ku JC, Alotaibi NM, Rutka JT. Retraction of Neurosurgical Publications: A Systematic Review. World Neurosurg [Internet]. 2017;103:809-814.e1. DOl: 10.1016/i.wneu.2017.04.014

11. Brainard J, You J. What a massive database of retracted papers reveals about science publishing's 'death penalty' [Internet]. Science. 2018 [cited 2020 Oct 17]. https://www.sciencemag.org/news/2018/10/what-massive-database-retracted-papers-revealsabout-science-publishing-s-death-penalty

12. Memon AR. Similarity and plagiarism in scholarly journal submissions: Bringing clarity to the concept for authors, reviewers and editors. J Korean Med Sci. 2020;35(27):1-8. DOI: 10.3346/jkms.2020.35.e217

13. Wang $T$, Xing QR, Wang $H$, Chen W. Retracted Publications in the Biomedical Literature from Open Access Journals. Sci Eng Ethics [Internet]. 2019;25(3):855-68. Available from: DOl: 10.1007/s11948-018-0040-6

14. Retraction Data Base [Internet]. [cited $2021 \quad$ Apr 10$].$ http://retractiondatabase.org/RetractionSearch.aspx

15. Mousavi T, Abdollahi M. A review of the current concerns about misconduct in medical sciences publications and the consequences. DARU, J Pharm Sci. 2020;28(1):359-69. DOI: 10.1007/s40199-020-00332-1

16. Nair S, Yean C, Yoo J, Leff J, Delphin E, Adams DC. Reasons for article retraction in anesthesiology: a comprehensive analysis. Can J Anesth [Internet]. 2020;67(1):57-63. https://doi.org/10.1007/s12630-019-01508-3

17. Marusic A, Wager E, Utrobicic A, Sambunjak D, Anderson MS, Rothstein HR. Interventions to prevent misconduct and promote integrity in research and publication. Cochrane Database Syst Rev. 2016;(4):1-81. DOI: 10.1002/14651858.MR000038.pub2

18. Yi N, Nemery B, Dierickx K. Integrity in Biomedical Research: A Systematic Review of Studies in China. Sci Eng Ethics [Internet]. 2019;25(4):1271-301. Available from: DOI: 10.1007/s11948018-0057-x

19. Stavale R, Ferreira GI, Galvão JAM, Zicker F, Novaes MRCG, de Oliveira CM, et al. Research misconduct in health and life sciences research: A systematic review of retracted literature from Brazilian institutions. PLoS One. 2019;14(4):1-17. DOI: 10.1371/journal.pone.0214272

20. Real Academia Española. Diccionario de la lengua española: definición de "plagiar" [Internet]. [cited 2020 Oct 17]. https://dle.rae.es/plagiar

21. Roig M. Encouraging editorial flexibility in cases of textual reuse. J Korean Med Sci. 2017;32(4):557-60.

22. Valpy FEJ. An etymological dictionary of the Latin language [Internet]. London: Baldwin and Co.; 1828. 345 p. https://archive.org/details/etymologicaldict00valp/page/n13/mode/2up

23. Martín SG. ¿Qué es plagio? Methodo Investig Apl a las Clencias Biol. 2020;5(4):171-4.

24. Antonio Aldrete J. Plagio y otros traspasos literario-científicos en medicina y particularmente en anestesiología. Rev Colomb Anestesiol. 2011;39(2). DOl: 10.5554/rca.v39i2.161

25. Mohammed RAA, Shaaban OM, Mahran DG, Attellawy HN, Makhlof A, Albasri A. Plagiarism in medical scientific research. J Taibah Univ Med Sci [Internet]. 2015;10(1):6-11. Available from: DOI: 10.1016/i.jtumed.2015.01.007

26. Sharma H, Verma S. Insight into modern-day plagiarism: The science of pseudo research. Tzu Chi Med J. 2020;32(3):240-4. DOI: 10.4103/tcmi.tcmi 21019

27. iThenticate. Research Ethics: Decoding Plagiarism and Attribution in Research [Internet]. [cited 2020 Oct 17]. https://cdn2.hubspot.net/hub/92785/file-318578964.pdf/ithenticate-decodingsurveysummary- 092413.pdf

28. Turnitin. Los 12 tipos de trabajo no original más comunes [Internet]. [cited 2021 Apr 10]. https://www.turnitin.com/es/infographics/prevencion-de-plagio

29. COPE $\mathrm{C}$ de E en P. COMITE DE ETICA EN PUBLICACIONES [Internet]. 2020 [cited 2020 Oct 15]. https://publicationethics.org 
30. The European Association of Science Editors (EASE). The European Association of Science Editors (EASE [Internet]. 2020 [cited 2020 Oct 15]. https://ease.org.uk

31. Roig M. Commentary: Ethical writing should be taught. Br Med J. 2006;333(7568):596-7. DOI: 10.1136/bmi.38946.501215.68

32. Memon AR, Mavrinac M. Knowledge, Attitudes, and Practices of Plagiarism as Reported by Participants Completing the AuthorAID MOOC on Research Writing. Sci Eng Ethics [Internet]. 2020;26(2):1067-88. DOI: 10.1007/s11948-020-00198-1

33. Mogull SA. Accuracy of cited "facts" in medical research articles: A review of study methodology and recalculation of quotation error rate. PLoS One. 2017;12(9):1-17. DOl: 10.1371/journal.pone.0184727

34. Masic I. Plagiarism and How to Avoid It. In: A Guide to the Scientific Career [Internet]. Wiley; 2019. p. 163-77. https://onlinelibrary.wiley.com/doi/abs/10.1002/9781118907283.ch19

35. Pezuk JA, Diniz SN, Pereira RM, Gonçalves ID, Costa NML da, Dias MA. El uso de softwares para identificar plagio en textos académicos y educacionales. Educ e Pesqui. 2020;46:1-22. DOI: 10.1590/S1678-4634202046217838

36. El Editor. En síntesis: ¿Cuánto debo plagiar para no ser acusado de plagiador? Form Univ. 2016;9(4):1. DOI: 10.4067/S0718-50062016000400001

37. ABC sociedad. El porcentaje nunca a va ser un criterio irrefutable para determinar si hay o no plagio en una tesis [Internet]. https://www.abc.es/sociedad/abci-porcentaje-nunca-criterioirrefutable-para-determinar-si-o-no-plagio-tesis-201809150323_noticia.html

38. Yip C, Han NLR, Sng BL. Legal and ethical issues in research. Indian J Anaesth. 2016;60(9):684-8. DOI: 10.4103/0019-5049.190627

39. Khadilkar SS. The Plague of Plagiarism: Prevention and Cure!!! J Obstet Gynecol India. 2018;68(6):425-31. DOI: 10.1007/s13224-018-1182-9

40. Bettaieb J, Cherif I, kharroubi G, Mrabet A. Attitudes towards plagiarism among academics of the faculty of Medicine of Tunis. Account Res [Internet]. 2020;27(8):521-37. Available from: DOl: 10.1080/08989621.2020.1780426

41. Chowhan A, Nandyala R, Patnayak R, Phaneendra B. Plagiarism: Trespassing the grey zone between searching and researching. Ann Med Health Sci Res. 2013;3(Suppl1):S56-8.

42. Masic I. The importance of proper citation of references in biomedical articles. Acta Inform Medica. 2013;21(3):148-55. https://www.ncbi.nlm.nih.gov/pmc/articles/PMC3853614/

43. Masic I, Hodzic A MS. Ethics in medical research and publication. Int J Prev Med. 2014;5(9):1073-82. https://www.ncbi.nlm.nih.gov/pmc/articles/PMC4192767/

44. Mašic I, Begić E, Donev DM, Gajović S, Gasparyan AY, Jakovljević M, et al. Sarajevo declaration on integrity and visibility of scholarly publications. Croat Med J. 2016;57(6):527-9.

45. Masic I, Donev D, Sinanovic O, Jakovljevic M, Zerem E, Milosevic DB, et al. The first mediterranean seminar on science writing, editing and publishing, Sarajevo, December 2-3, 2016. Acta Inform Medica. 2016;24(6):424-35. DOI: 10.5455/aim.2016.24.424-435

46. Masic I. The Second Mediterranean Seminar on Science Writing, Editing and Publishing (SWEP2018), Sarajevo, December 8th, 2018. Med Arch (Sarajevo, Bosnia Herzegovina). 2018;72(6):461-5.

47. (OSA) OS of A. Publishing.From the Board of Editors: on Plagiarism [Internet]. 2020 [cited 2020 Oct 15]. https://www.osapublishing.org/submit/review/plagiarism_2013.pdf

48. Morton NS. Publication ethics. Paediatr Anaesth. 2009;19(10):1011-3.

49. Siva N. Plagiarism: Cutting out cut-and-paste jobs. Nat Clin Pract Urol. 2008;5(7):351.DOI: 10.1038/ncpuro1169

50. Sanabria L. Conceptualización jurídica del plagio en Colombia. Rev Colomb Cirugía. 2014;29(2):88-97. https://www.revistacirugia.org/index.php/cirugia/article/view/417 
51. Alvarez A. JC, Ceballos Bedoya MA, Muñoz Sierra ÁM. De los delitos contra los derechos de autor en el Código Penal colombiano. Nuevo Foro Penal. 2015;9(81):93-115. DOl: 10.17230/nfp.9.81.3

52. Masic I. Plagiarism in Scientific Research and Publications and How to Prevent It. Mater Socio Medica. 2014;26(2):141. https://www.ncbi.nlm.nih.gov/pmc/articles/PMC4035147/

53. Gunnarsson J, Kulesza WJ, Pettersson A. Teaching International Students How to Avoid Plagiarism: Librarians and Faculty in Collaboration. J Acad Librariansh [Internet]. 2014;40(34):413-7. DOI: 10.1016/i.acalib.2014.04.006

54. Schuster E, Levkowitz H, Oliveira O, Aluisio S, Dayrell C, Feltrim V, Tagnin S Z V. Writing Scientific Papers in English Successfully: your complete roadmap. First Edit. Schuster E, Levkowitz H OO, editor. Sao Carlos, Brazil: Compacta Grafica e Editora; 2014. 1-192 p. 